\title{
Chemical enrichment and accretion of nitrogen-loud quasars ${ }^{\star \star \star}$
}

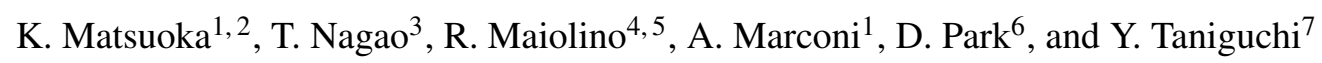 \\ 1 Dipartimento di Fisica e Astronomia, Universitá degli Studi di Firenze, via G. Sansone 1, 50019 Sesto Fiorentino, Italy \\ e-mail: matsuoka@arcetri.astro.it \\ 2 INAF-Osservatorio Astrofisico di Arcetri, Largo Enrico Fermi 5, 50125 Firenze, Italy \\ 3 Research Center for Space and Cosmic Evolution, Ehime University, 2-5 Bunkyo-cho, 790-8577 Matsuyama, Japan \\ 4 Cavendish Laboratory, University of Cambridge, 19 J. J. Thomson Avenue, Cambridge CB3 0HE, UK \\ 5 Kavli Institute for Cosmology, University of Cambridge, Madingley Road, Cambridge CB3 OHA, UK \\ 6 Korea Astronomy and Space Science Institute, 776 Daedeok-daero, Yuseong-gu, 34055 Daejeon, Republic of Korea \\ 7 The Open University of Japan, 2-11 Wakaba, Mihama-ku, 261-8586 Chiba, Japan
}

Received 11 October 2016 / Accepted 18 September 2017

\begin{abstract}
We present rest-frame optical spectra of 12 "nitrogen-loud" quasars at $z \sim 2.2$, whose rest-frame ultraviolet (UV) spectra show strong nitrogen broad emission lines. To investigate their narrow-line region (NLR) metallicities, we measure the equivalent width (EW) of the [O III] $] 5007$ emission line: if the NLR metallicity is remarkably high, as suggested by the strong UV nitrogen lines, the $[\mathrm{O}$ III $] \lambda 5007$ line flux should be very weak due to the low equilibrium temperature of the ionized gas owing to significant metal cooling. In the result we found that our spectra show moderate EW of the [O III] $\lambda 5007$ line similar to general quasars. This indicates that nitrogen-loud quasars do not have extremely metal-rich gas clouds in NLRs. This suggests that strong nitrogen lines from broadline regions (BLRs) originate from exceptionally high abundances of nitrogen relative to oxygen without very high BLR metallicities. This result indicates that broad emission lines of nitrogen are not good indicators of the BLR metallicity in some cases. On the other hand, we also investigate virial black hole masses and Eddington ratios by using the $\mathrm{H} \beta$ and $\mathrm{C}$ IV $\lambda 1549$ lines for our sample. As a result, we found that black hole masses and Eddington ratios of nitrogen-loud quasars tend to be low and high relative to normal quasars, suggesting that nitrogen-loud quasars seem to be in a rapidly accreting phase. This can be explained in terms of a positive correlation between Eddington ratios and nitrogen abundances of quasars, which is probably caused by the connection between the mass accretion onto black holes and nuclear star formation.
\end{abstract}

Key words. galaxies: active - galaxies: nuclei - quasars: emission lines - quasars: general

\section{Introduction}

Chemical evolution is one of the most crucial topics in extragalactic research because metal formations and its enrichments have close connections with the star formation in galaxies, corresponding to the galaxy evolution. The simplest way to investigate the chemical evolution of galaxies is by measuring metallicities or chemical abundances of galaxies at various redshifts and exploring its redshift evolution. Observationally, the gas-phase metallicity of star-forming galaxies in the local Universe can be measured by using optical emission-line spectra, e.g., [O II] $] \lambda 3727, \mathrm{H} \beta,[\mathrm{O} \mathrm{III}] \lambda 4959,[\mathrm{O} \mathrm{III}] \lambda 5007, \mathrm{H} \alpha$, and [N II] $\lambda 6584$ (see Pagel et al. 1979; Kewley \& Dopita 2002; Nagao et al. 2006a; Ly et al. 2016, and references therein), and even at $z>1$ current near-infrared observations can cover these lines (e.g., Maiolino et al. 2008; Wuyts et al. 2016), with some caveats such as systematic errors in the calibration (see, e.g., Kewley \& Ellison 2008). However, at $z>3$, these diagnostics are more difficult because these emission lines are redshifted out of near-infrared wavelengths.

\footnotetext{
$\star$ Based on observations collected at the European Organisation for Astronomical Research in the Southern Hemisphere under ESO programme 088.B-0191(A), and at the Subaru Telescope, which is operated by the National Astronomical Observatory of Japan.

$\star \star$ Reduced spectra (FITS files) are available at the CDS via anonymous ftp to cdsarc.u-strasbg. fr (130.79.128.5) or via http://cdsarc.u-strasbg.fr/viz-bin/qcat?J/A+A/608/A90
}

An alternative way to measure metallicities of galaxies in the early Universe is by focusing on the active Galactic nucleus (AGN). In fact, this approach has two main advantages in the high- $z$ research. First, thanks to its huge luminosity we can obtain high quality spectra even at high redshift. Second, because of various emission lines shown in rest-frame ultraviolet (UV) wavelength we can extract chemical properties from observed-frame optical spectra. The prominent emission-line spectra of AGNs provide chemical properties of gas clouds illuminated by the central engine: it is possible to investigate chemical properties, even in the Galactic scale, if we focus on emission lines emitted from the narrow-line regions (NLRs) $\left(R_{\mathrm{NLR}} \sim 10^{2-4} \mathrm{pc}\right.$; e.g., Bennert et al. 2006a,b), instead of broad-line regions (BLRs). Thus by using AGNs, we can reveal the chemical evolution in the early Universe, closely related to the formation and evolution of galaxies. In addition, we emphasize that only AGNs allow us to estimate black hole masses in the high- $z$ Universe based on the kinematical state of BLR clouds. Therefore, AGN spectra are useful also in order to understand the co-evolution of galaxies and supermassive black holes (SMBHs) in the high- $z$ epoch in addition to the chemical evolution.

In the diagnostics of AGN metallicities, we often recognize that nitrogen is one of the most useful elements since it is a secondary element and its abundance is expected to be proportional to the square of the metallicity, i.e., $\mathrm{N} / \mathrm{H} \propto(\mathrm{O} / \mathrm{H})^{2}$. The spectra of AGN basically show some broad emission lines of nitrogen, 
e.g., N V $\lambda 1240$, N IV] $\lambda 1486$, and N III] $\lambda 1749$, in the UV wavelength. Hamann \& Ferland $(1992,1993)$ used emission-line flux ratios of $\mathrm{N} V \lambda 1240 / \mathrm{C}$ IV $\lambda 1549$ and $\mathrm{N}$ V $\lambda 1240 / \mathrm{He}$ II $\lambda 1640$ as indicators of the BLR metallicity (see also Hamann \& Ferland 1999; Hamann et al. 2002). Subsequently, many papers follow this method based on nitrogen lines to investigate BLR metallicities (e.g., Warner et al. 2004; Nagao et al. 2006b; Dietrich et al. 2009; Matsuoka et al. 2011; Shin et al. 2013).

In previous studies, emission-line flux ratios including nitrogen lines have mainly been used to estimate the BLR metallicity, $Z_{\mathrm{BLR}}$, as described above. Based on this method, it has been found that the BLR in the most luminous quasars show supersolar metallicities (e.g., Hamann \& Ferland 1992; Dietrich et al. 2003; Nagao et al. 2006b), even for quasars at $z>6$ (e.g., Kurk et al. 2007; Jiang et al. 2007; Juarez et al. 2009; Mortlock et al. 2011). Moreover, Osmer \& Smith (1980) discovered an unusual quasar, Q0353-383, at $z=1.96$ that has prominent broad emission lines of $\mathrm{N}$ III] $\lambda 1749, \mathrm{~N}$ IV] $\lambda 1486$, and N V $\lambda 1240$ : this is called the nitrogen-loud quasar (hereafter $\mathrm{N}$-loud quasar). Baldwin et al. (2003b) claimed that these unusual nitrogen emissions in Q0353-383 are likely due to high metallicity in the BLR; the metallicity estimated by these nitrogen lines reaches $\sim 15 Z_{\odot}$ (see also Osmer 1980) if we assume the relation of $\mathrm{N} / \mathrm{H} \propto Z_{\mathrm{BLR}}^{2}$. However, we note that such extremely high metallicity is very hard to reconcile with galaxy chemical evolutionary models. Therefore, the N-loud quasars raise a question whether or not the broad nitrogen lines are really reliable for estimating $Z_{\mathrm{BLR}}$.

Motivated by this question, the rest-frame UV spectrum of $\mathrm{N}$-loud quasars has been investigated. By using $293 \mathrm{~N}$-loud quasars selected from the Sloan Digital Sky Survey (SDSS; York et al. 2000), Jiang et al. (2008) reported that N-loud quasars may not have such high BLR metallicities, but may just have unusually high relative abundances of nitrogen because their rest-frame UV spectra are not significantly different from those of typical normal quasars, except for the nitrogen lines; this means that metallicity-sensitive emission line flux ratios excluding nitrogen do not show the extremely high metallicity expected from nitrogen lines. This explanation throws a doubt on the use of nitrogen lines for BLR metallicity measurements for quasars, at least in some cases. On the other hand, Batra \& Baldwin (2014) aimed to clarify this controversial point by estimating BLR metallicities of $\mathrm{N}$-loud quasars, and claimed that strong nitrogen lines of N-loud quasars are caused by high BLR metallicities and not the high relative abundance of nitrogen. We note, however, that since this study was concluded only by using emission line flux ratios including nitrogen lines, i.e., $\mathrm{N}$ V $\lambda 1240, \mathrm{~N}$ IV] $\lambda 1486$, and $\mathrm{N}$ III] $\lambda 1750$, it would be still unclear whether N-loud quasars have extremely metal-rich gas clouds in BLRs: we need to estimate BLR metallicities by using emission line flux ratios including nitrogen lines and also flux ratios excluding nitrogen lines, and comparing them with each other.

To solve this problem regarding the origin of strong nitrogen lines seen in quasar spectra, we focus on NLR metallicities instead of BLR ones. If BLR metallicities of N-loud quasars are extremely high, NLR values would be also relatively high, although both absolute values are not the same (e.g., Fu \& Stockton 2007; Du et al. 2014). Recently, Araki et al. (2012) investigated the NLR metallicity of a N-loud quasar at $z=3.16$, SDSS J1707+6443, by using the [O III] $\lambda 5007$ emission line shown in rest-frame optical wavelengths: if the NLR metallicity is remarkably high as suggested by strong UV nitrogen lines, the [O III] $\lambda 5007$ line flux should be very weak due to the low equilibrium temperature of the ionized gas owing to significant metal cooling. We also examined its other parameters (e.g., ionization parameter and hydrogen density of NLR

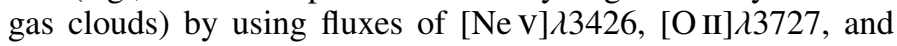
[Ne III] $\lambda 3869$, which are used to correctly estimate the NLR metallicity. In the result we found that the NLR in this N-loud quasar is not extremely metal rich, which is not consistent with the naive expectation from unusually strong nitrogen broad lines. We note, however, that it is difficult to conclude that the N-loud quasar is not extremely metal rich generally because this result was obtained only from one object. Therefore, additional data sets of rest-frame optical spectra of N-loud quasars are crucially needed to investigate whether or not BLR metallicities of N-loud quasars are universally extremely high.

Furthermore, if N-loud quasars do not have extremely high metallicities as Araki et al. (2012) claimed, unusually high nitrogen abundance relative to oxygen is required. Since the chemical properties of gas in Galactic nuclei are closely related to the past star formation history and the gas radial motion, we focus on the relation between the mass accretion to SMBHs and the nuclear star formation. Recently, by using optical spectra of 2383 SDSS quasars at $2.3<z<3.0$, Matsuoka et al. (2011) investigated the dependence of the emission-line flux ratios (BLR metallicity indicators) on the Eddington ratio. They found that the Eddington ratio depends on the emission-line flux ratios involving $\mathrm{N} v \lambda 1240$, while it does not correlate with the other emission-line flux ratios without the nitrogen line (see Fig. 6 in Matsuoka et al. 2011). This result means that the relative nitrogen abundance seems to depend on the Eddington ratio. This indicates that a rapid mass accretion onto SMBHs is associated with a post-starburst phase when asymptotic giant branch (AGB) stars enrich the interstellar medium with nitrogen. By considering this relation, we can obtain one possible scenario that N-loud quasars are in an AGN active phase, i.e., a high accretion phase. This would be helpful in order to understand the connection between AGN and star formation activities, closely related to the co-evolution of galaxies and SMBHs. In this sense, therefore, it is also important to examine whether N-loud quasars show high Eddington ratios.

In this paper, we present near-infrared spectra of $12 \mathrm{~N}$-loud quasars and investigate their spectroscopic properties. Specifically, we estimate NLR metallicities, black hole masses, and Eddington ratios by using emission lines in the rest-frame optical wavelength. In Sect. 2, we describe our sample of N-loud quasars and observations. Section 3 shows the spectral measurements. We summarize our results in Sect. 4 , and discuss the interpretation of results in Sect. 5. The summary is given in Sect. 6. Here, we adopt a concordance cosmology with $\left(\Omega_{\mathrm{M}}, \Omega_{\Lambda}\right)=(0.3,0.7)$ and $H_{0}=70 \mathrm{~km} \mathrm{~s}^{-1} \mathrm{Mpc}^{-1}$.

\section{Observations and data reduction}

\subsection{Target selection}

We select our targets from the N-loud quasar catalogue of Jiang et al. (2008). They collected 293 N-loud quasars from the SDSS Fifth Data Release (Schneider et al. 2007) with the following criteria: (1) rest-frame equivalent widths (EW) of N IV] $\lambda 1486$ or N III] $\lambda 1750>3 \AA$ which are significantly higher than those of normal quasars, i.e., $\sim 0.4 \AA$ for $\mathrm{N} \mathrm{III]}] \lambda 1750$ line and typically no detection for N IV] $\lambda 1486$ line (see also Vanden Berk et al. 2001); (2) the redshift range $1.7<z<4.0$ covering one or two broad nitrogen lines in SDSS optical spectra; and (3) $i^{\prime}<20.1$. To obtain emission lines of [O III] $\lambda 5007$, $\mathrm{H} \beta$, [Ne III] $\lambda 3869$, [O II] $\lambda 3727$, and [Ne V] 33426 in the nearinfrared wavelength range, in this study we select 12 quasars at 
Table 1. Journal of spectroscopic observations.

\begin{tabular}{ccccccc}
\hline \hline Target & RA & Dec & $z^{a}$ & $i^{b}$ & Instrument & Date \\
\hline SDSS J0357-0528 & 035722.94 & -052837.1 & 2.2887 & 19.09 & Subaru/IRCS & 23 Jan. 2012 \\
SDSS J0931+3439 & 093152.76 & +343920.6 & 2.3051 & 19.44 & Subaru/IRCS & 23 Jan. 2012 \\
SDSS J1211+0449 & 121113.56 & +044946.2 & 2.3184 & 18.87 & Subaru/IRCS & 23 Jan. 2012 \\
SDSS J0756+2054 & 075627.70 & +205413.9 & 2.2109 & 19.11 & NTT/SOFI & 4 Feb. 2012 \\
SDSS J0803+1742 & 080325.04 & +174239.0 & 2.0924 & 18.25 & NTT/SOFI & 3 Feb. 2012 \\
SDSS J0857+2636 & 085750.12 & +263634.3 & 2.1394 & 18.09 & NTT/SOFI & 6 Feb. 2012 \\
SDSS J0938+0900 & 093801.18 & +090004.4 & 2.1593 & 18.05 & NTT/SOFI & 5 Feb. 2012 \\
SDSS J1036+1247 & 103638.14 & +124745.9 & 2.1557 & 18.66 & NTT/SOFI & 5 Feb. 2012 \\
SDSS J1110+0456 & 111048.93 & +045608.0 & 2.2077 & 18.82 & NTT/SOFI & 3 Feb. 2012 \\
SDSS J1130+1512 & 113036.42 & +151253.1 & 2.2271 & 18.56 & NTT/SOFI & 5 Feb. 2012 \\
SDSS J1134+1500 & 113423.00 & +150059.2 & 2.1078 & 18.68 & NTT/SOFI & 6 Feb. 2012 \\
SDSS J1308+1136 & 130811.96 & +113609.2 & 2.1012 & 17.70 & NTT/SOFI & 5 Feb. 2012 \\
\hline
\end{tabular}

Notes. ${ }^{(a)}$ Redshift from Shen et al. (2008); ${ }^{(b)}$ SDSS $i$-band magnitude corrected for Galactic extinction in Jiang et al. (2008); ${ }^{c}$ observed only with $H$ band.

$2.09<z<2.57$ from the catalogue with the criteria of observable coordinates and apparent magnitudes given in Table 1.

\subsection{Near-infrared spectroscopic observations}

We observed our targets by using the Infrared Camera and Spectrograph (IRCS; Kobayashi et al. 2000) with the adaptive optics system with 188 elements (AO188; Hayano et al. 2008, 2010) on the Subaru Telescope, and with the Son of ISAAC (SOFI; Moorwood et al. 1998) on the New Technology Telescope (NTT). The observational logs are given in Table 1.

For the IRCS observation, we obtained $J$ - and $H$-band spectra of three targets at $z \sim 2.3$ by using a $0^{\prime \prime} .225$ slit. Thanks to AO188 with the laser guide star (LGS) mode, we were able to achieve typical point-spread function sizes of $\sim 0.25^{\prime \prime}$ around $13000 \AA$ and $\sim 0.21^{\prime \prime}$ at $16000 \AA$. In the $H$-band observation, we measured the emission lines of $[\mathrm{O}$ III $] \lambda 5007$ and $\mathrm{H} \beta$, whose expected wavelength is $16000 \AA<\lambda_{\text {obs }}<16600 \AA$, to investigate NLR metallicities and black hole masses. On the other hand, in the $J$-band observation we focused on the emission lines of $[\mathrm{O} \mathrm{II}] \lambda 3727$ and $[\mathrm{Ne} \mathrm{III}] \lambda 3869$, redshifted to the range at $12200 \AA<\lambda_{\text {obs }}<12800 \AA$. These emission lines are used to investigate properties of NLR gases (e.g., ionization parameters and hydrogen densities of clouds). The spectral dispersion is $\sim 4.68 \AA$ pixel $^{-1}$ and the spectral resolution estimated from $\mathrm{OH}$ emission lines was $\sim 1000$. The total exposure time for each target was roughly $7000 \mathrm{~s}$ for $J$ band and $2500 \mathrm{~s}$ for $H$ band. The unit exposure times were 300-600 s.

The SOFI observations were performed with the Grism Blue Filter $(\mathrm{GBF})$ to cover the wavelength at $9500 \AA<\lambda_{\text {obs }}<$ $16400 \AA$, where we were able to cover five emission lines of the nine remaining $\mathrm{N}$-loud quasars at $2.1 \lesssim z \lesssim 2.2$ simultaneously. Typical seeing size was $\sim 1.5^{\prime \prime}$. We used a long slit, $0^{\prime \prime}$.6 in width, and the dispersion was $\sim 6.93 \AA$ pixel $^{-1}$. The spectral resolution was $\sim 650$. Total exposure times were $\sim 7200 \mathrm{~s}$ for each target, adopting an unit exposure time of $\sim 600 \mathrm{~s}$.

\subsection{Data reduction}

For all spectra, standard data reduction procedures were performed using available IRAF ${ }^{1}$ tasks. By using dome-flat frames,

\footnotetext{
1 IRAF is distributed by the National Optical Astronomy Observatory, which is operated by the Association of Universities for Research in Astronomy (AURA) under a cooperative agreement with the National Science Foundation.
}

object frames were flat-fielded and thus the first sky subtraction was performed with the A-B method; we subtracted pairs of subsequent frames with the target at different positions along the slit. Cosmic ray events were removed by using the lacos_spec task (van Dokkum 2001). We extracted the one-dimensional spectra with the apall task, using an 8-pixel $\left(\sim 0.4^{\prime \prime}\right)$ aperture for IRCS and 11-pixel $\left(\sim 3.0^{\prime \prime}\right)$ for SOFI. In this extraction, we fit the residuals of sky background and subtracted them. The wavelength calibration was executed with arc lamp frames. Finally, flux calibration and telluric absorption correction were carried out by using observed spectra of telluric standard stars. We took defocused data for bright standard star observations to avoid the saturation of the detector. This step affected the flux calibration: the obtained fluxes were overestimated due to the extensive slit loss of the defocused data.

To correct observational uncertainties, e.g., the slit loss, mainly in observations of standard stars and weather condition, we re-calibrated fluxes by using photometric magnitudes of the targets themselves. We obtained near-infrared magnitudes of the $J$ and $H$ bands from $i$-band magnitude using the color- $z$ relation of SDSS-UKIDSS quasars in Wu \& Jia (2010) since there is almost no near-infrared magnitude for our targets. We note that only two objects (SDSS J0938+0900 and SDSS J1308+1136) have been observed with $J$ and $H$ bands through the United Kingdom Infrared Telescope (UKIRT) Infrared Deep Sky Survey (UKIDSS; Lawrence et al. 2007), and we confirmed that these observed magnitudes are consistent with the estimated values describe above with uncertainties of $\sim 2 \%$. By comparing the extrapolated photometric magnitudes with spectroscopic magnitudes measured from our spectra, we scaled fluxes of the spectra; the mean value of the scaling factor is $\sim 0.8$. The final reduced spectra are shown in Fig. 1.

\section{Spectral measurements}

To measure line properties, we fit emission lines of $\mathrm{H} \beta$ and [O III $] \lambda 4959,5007$ lines with Gaussian functions. In the multi-component fitting processes, we used a routine, mpcurvefit, in the Interactive Data Language (IDL). First, we converted our observed spectra to the rest frame. Second, we fit the continuum with a single power-law model. In this fitting, we ignored the host-galaxy starlight and the Fe II emission blends due to low signal-to-noise ratios, $\mathrm{S} / \mathrm{N}$. The best 

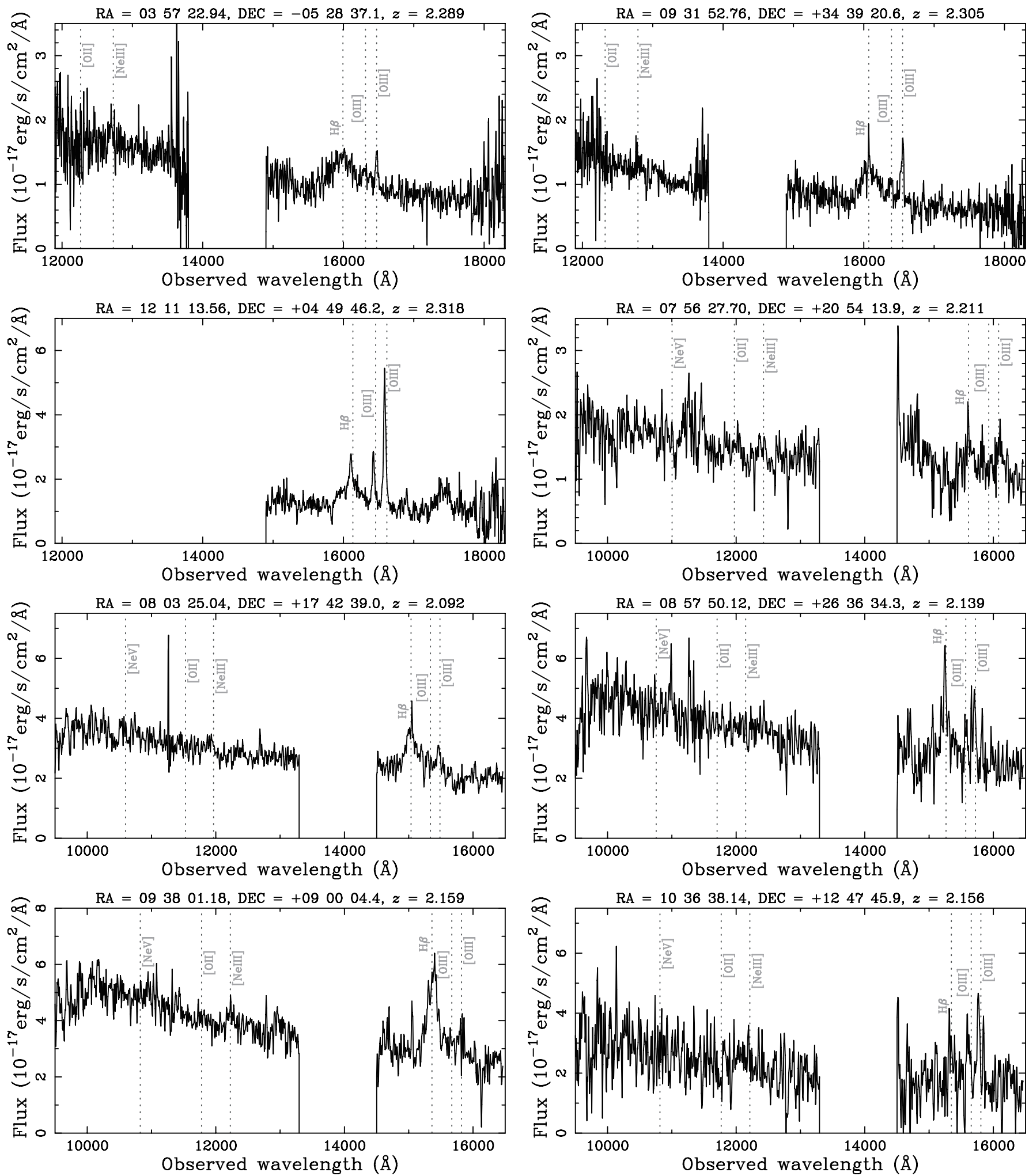

Fig. 1. Near-infrared spectra of N-loud quasars obtained in our IRCS and SOFI runs, adopting the two-pixel binning in the wavelength direction. Gray dotted lines show the wavelengths of some rest-frame optical emission lines, i.e., [Ne V] $\lambda 3426,[\mathrm{O}$ II] $] 33727,[\mathrm{Ne}$ III] $\lambda 3869, \mathrm{H} \beta$, [O III] $\lambda 4959$, and [O III] $\lambda 5007$ lines, expected from redshifts given in Shen et al. (2008).

continuum fit was determined based on the $\chi^{2}$ statistics in the wavelength ranges where there are no strong emission lines, e.g., 4600-4750 $\AA$ and 5050-5150 $\AA$ in the rest frame. From the power-law continuum model, we estimate the monochromatic luminosity at $5100 \AA$.
After the pseudo-continuum model was subtracted from the original spectrum, we fit the emission of the $\mathrm{H} \beta$, [O III] $\lambda 4959$, and $[\mathrm{O}$ III $] \lambda 5007$ lines. We used one or two Gaussians for the broad and narrow $\mathrm{H} \beta$ components. For the narrow [O III] $\lambda 4959$ and [O III $] \lambda 5007$ lines, we adopted the single-Gaussian fitting; their flux ratio is fixed as three. For all narrow components, we 

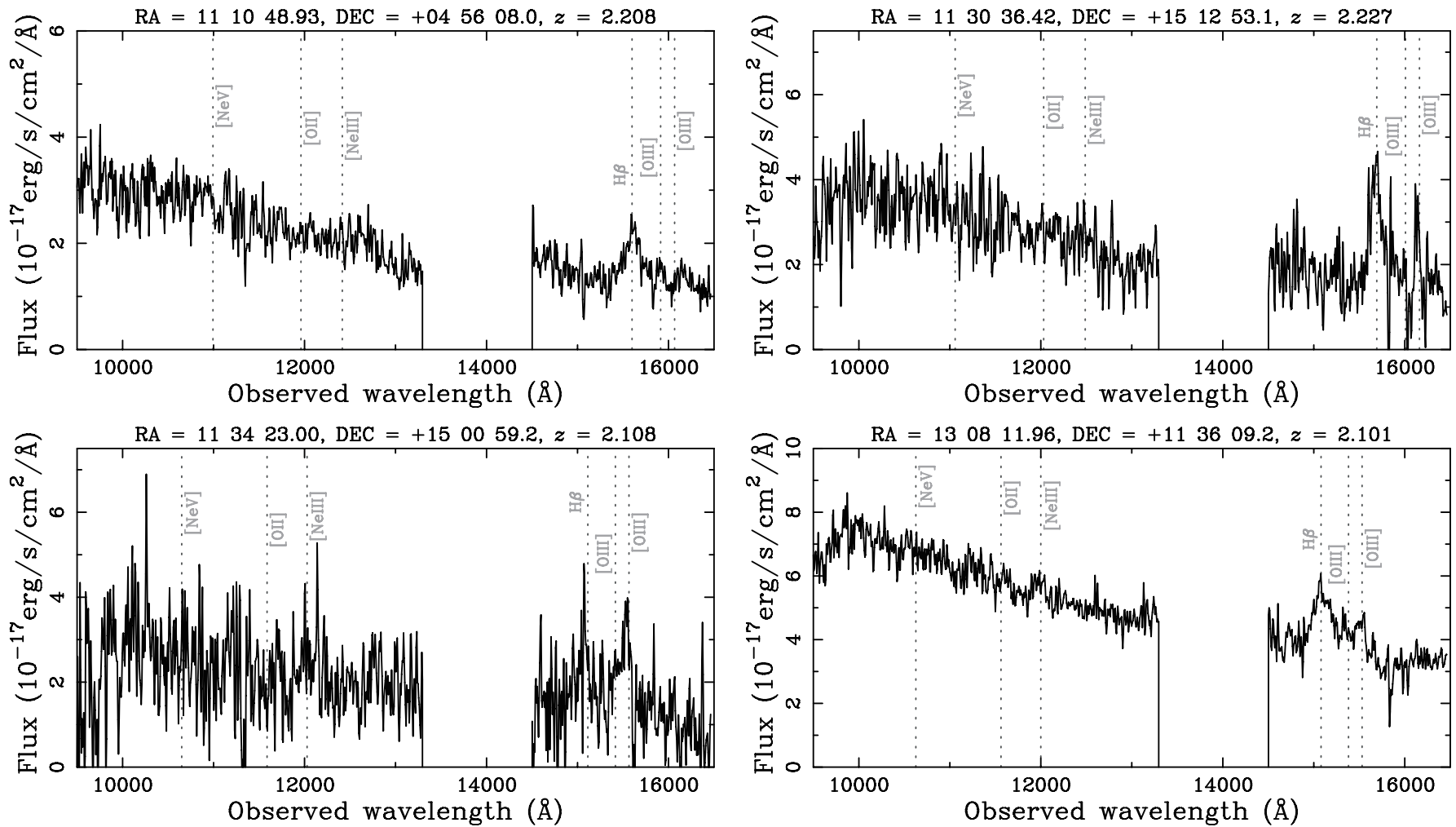

Fig. 1. continued.
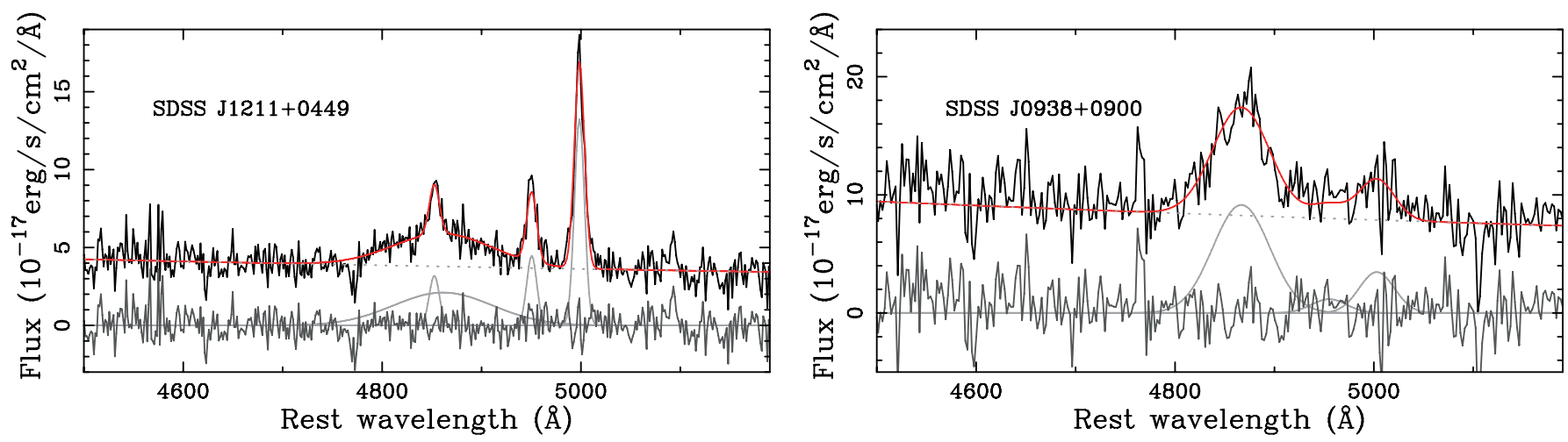

Fig. 2. Examples of spectral fittings in rest-frame optical spectra of two N-loud quasars (SDSS J1211+0449 and SDSS J0938+0900). Red lines show total fitting results. Gray dotted lines denote fits of continuum, and individual emission-line components are indicated as light gray Gaussian curves. Dark gray lines denote the residuals.

adopted the same velocity shift and width by assuming the same kinematics of NLR clouds. We measured the emission-line flux of $[\mathrm{O} \mathrm{III}] \lambda 5007$ and obtained the rest-frame equivalent width, $\mathrm{EW}_{[\mathrm{O} \text { III] }}$, with the continuum flux at $5007 \AA$ from the power-law model.

To estimate uncertainties of the line width and luminosity measurements, we adopted the Monte Carlo flux randomization method (e.g., Bentz et al. 2009; Shen et al. 2011; Park et al. 2012). We generated 1000 mock spectra for each observed spectrum by adding Gaussian random noise based on the flux errors. Then, we measured the line widths and AGN luminosities from the simulated spectra. We derived the standard deviation of their distribution as the measurement uncertainty. We also gave $3 \sigma$ upper limits for the [Ne v] 33426 , [O II] $] 3727$, and [Ne III] $\lambda 3869$ lines by adopting the average velocity width of the
[O III] $\lambda 5007$ emission line ( $\Delta v \sim 1450 \mathrm{~km} \mathrm{~s}^{-1}$ ) since we could not detect these emission lines in our observations (see Fig. 1).

In Fig. 1, some objects (e.g., SDSS J1211+0449 and SDSS $\mathrm{J} 1036+1247$ ) seem to show blueshifted narrow lines compared to the expected central wavelength indicated by dotted vertical lines (Shen et al. 2008). Although these velocity shifts may be a sign of gas outflow (e.g., Rupke et al. 2005; Bae \& Woo 2014; Woo et al. 2016; Bae et al. 2017), the accuracy of the redshift measurement needs to be considered carefully in order to discuss this topic, and this is beyond the scope of this paper. We note that this uncertainty of redshift does not affect our conclusion.

Figure 2 shows two examples of the fitting results. Measurement results, i.e., line fluxes and equivalent widths of the [O III] $\lambda 5007$ line, the full widths at half maximum (FWHM) of the broad $\mathrm{H} \beta$ line, and monochromatic luminosities at $5100 \AA$, 
Table 2. Results of spectral fitting.

\begin{tabular}{|c|c|c|c|c|c|c|}
\hline Object & $\begin{array}{c}F_{[\mathrm{O} \mathrm{III}]} \\
{\left[10^{-17} \mathrm{erg} \mathrm{s}^{-1} \mathrm{~cm}^{-2}\right]}\end{array}$ & $\begin{array}{c}\log E W_{[\mathrm{O} \mathrm{IIII}}{ }^{a} \\
{[\AA]} \\
\end{array}$ & $\begin{array}{c}\log F W H M_{\mathrm{H} \beta}{ }^{b} \\
{\left[\mathrm{~km} \mathrm{~s}^{-1}\right]}\end{array}$ & $\begin{array}{c}\log \left(\lambda L_{5100}\right) \\
{\left[\operatorname{erg~s}^{-1}\right]} \\
\end{array}$ & $\begin{array}{c}\log M_{\mathrm{BH}, \mathrm{H} \beta}{ }^{c} \\
{\left[M_{\odot}\right]}\end{array}$ & $\log \left(L / L_{\mathrm{Edd}}\right)_{\mathrm{H} \beta}^{c}$ \\
\hline 8 & $28.19 \pm 4.80$ & $880 \pm 0.205$ & $3.953 \pm 0.014$ & $45.768 \pm 0.001$ & $9.699 \pm 0.028$ & - \\
\hline & & & & & 28 & \\
\hline SDSS J12 & $6.49 \pm 9.27$ & & 014 & 45.873 & $9.502 \pm 0$ & .029 \\
\hline 054 & $.31 \pm 14.89$ & & .050 & 45.775 & $9.006 \pm$ & -0 \\
\hline SD & 48 & & & 1 & & 028 \\
\hline SD & 1.39 & & & & 9. & \\
\hline & .67 & & 14 & 46 & 9.2 & -0 \\
\hline SDSS J1036+1247 & $6.64 \pm 21.00$ & $1.583 \pm 0.328$ & $3.208 \pm 0.041$ & $45.899 \pm 0.007$ & $8.275 \pm 0.081$ & $0.490 \pm 0.082$ \\
\hline & & & $3.640 \pm 0.013$ & $45.822 \pm 0.001$ & $9.102 \pm 0.026$ & $-0.413 \pm 0.026$ \\
\hline & $103.62 \pm 26.12$ & $1.501 \pm$ & & & $8.752 \pm$ & 018 \\
\hline & $222.87 \pm 57.56$ & $1.747 \pm 0$ & 030 & $45.809 \pm$ & $8.964 \pm 0.061$ & $-0.288 \pm 0.061$ \\
\hline SDSS J1308+1136 & $165.45 \pm 30.19$ & $1.204 \pm 0.193$ & $3.858 \pm 0.019$ & $46.220 \pm 0.002$ & $9.737 \pm 0.037$ & $-0.650 \pm 0.037$ \\
\hline
\end{tabular}

Notes. ${ }^{(a)}$ Logarithmic rest-frame equivalent widths of the [O III] $\lambda 5007$ line; ${ }^{(b)}$ logarithmic rest-frame line widths of the $\mathrm{H} \beta$ broad line after the correction for instrument broadening; ${ }^{(c)}$ only statistical errors are given instead of systematic ones; ${ }^{(d)} 3 \sigma$ upper limits given for the [O III] $\lambda 5007$ undetected object.

Table 3. Estimates of $3 \sigma$ flux upper limits of undetected emission lines.

\begin{tabular}{cccc}
\hline \hline Object & $\begin{array}{c}F_{[\mathrm{Ne}]} \\
{\left[10^{-17}\right.}\end{array}$ & $\begin{array}{c}F_{[\mathrm{OII}]} \\
\mathrm{erg} \mathrm{s}^{-1}\end{array}$ & $\begin{array}{c}F_{[\mathrm{Ne} \text { III }} \\
\left.\mathrm{cm}^{-2}\right]\end{array}$ \\
\hline SDSS J0357-0528 & $-^{a}$ & $<16.47$ & $<9.41$ \\
SDSS J0931+3439 & $-^{a}$ & $<10.43$ & $<6.40$ \\
SDSS J1211+0449 & $-^{a}$ & $-^{a}$ & $-^{a}$ \\
SDSS J0756+2054 & $<17.23$ & $<12.57$ & $<14.52$ \\
SDSS J0803+1742 & $<17.89$ & $<13.29$ & $<12.95$ \\
SDSS J0857+2636 & $<11.75$ & $<7.15$ & $<10.77$ \\
SDSS J0938+0900 & $<28.46$ & $<18.74$ & $<24.54$ \\
SDSS J1036+1247 & $<21.31$ & $<15.20$ & $<14.71$ \\
SDSS J1110+0456 & $<14.39$ & $<8.80$ & $<11.52$ \\
SDSS J1130+1512 & $<16.26$ & $<9.87$ & $<14.31$ \\
SDSS J1134+1500 & $<49.02$ & $<46.02$ & $<42.98$ \\
SDSS J1308+1136 & $<29.67$ & $<29.45$ & $<16.50$ \\
\hline
\end{tabular}

Notes. ${ }^{(a)}$ Out of the wavelength coverage; ${ }^{(b)}$ based on the averaged $\mathrm{FWHM}_{[\mathrm{OIII}]}$ value of $24.2 \AA$, due to the lack of the [O III] $\lambda$ 5007 line detection.

are listed in Table 2, and $3 \sigma$ upper limits of fluxes for the undetected emission lines, i.e., [Ne v] 33426 , [O II] $] \lambda 3727$, and [Ne III] $\lambda 3869$ lines, are shown in Table 3.

\section{Results}

As described in Sect. 2, we obtained near-infrared (i.e., $J$ - and $H$ bands) spectra of $12 \mathrm{~N}$-loud quasars as shown in Fig. 1. In $J$-band wavelength, there is no significant detection of emission lines, e.g., [Ne III] $\lambda 3869$, as Araki et al. (2012) found in the spectra of SDSS J1707+6443. On the other hand, $H$-band spectra show significant detections of $\mathrm{H} \beta$, [O III] $] 4959$, and [O III] $\lambda 5007$ lines for most of the objects in our sample.

Figure 3 shows distributions of observed rest-frame equivalent widths of the [O III] $\lambda 5007$ line of N-loud quasars, denoted as black-line filled histograms. Here, we added SDSS J1707+6443 in Araki et al. (2012) to our sample. In order to compare these $\mathrm{N}$-loud quasars with general quasars, we also plot [O III] $\lambda 5007$ equivalent widths of SDSS quasars at $0<z<1$ (Shen et al. 2011), shown as gray-line open histograms in Fig. 3. Moreover,

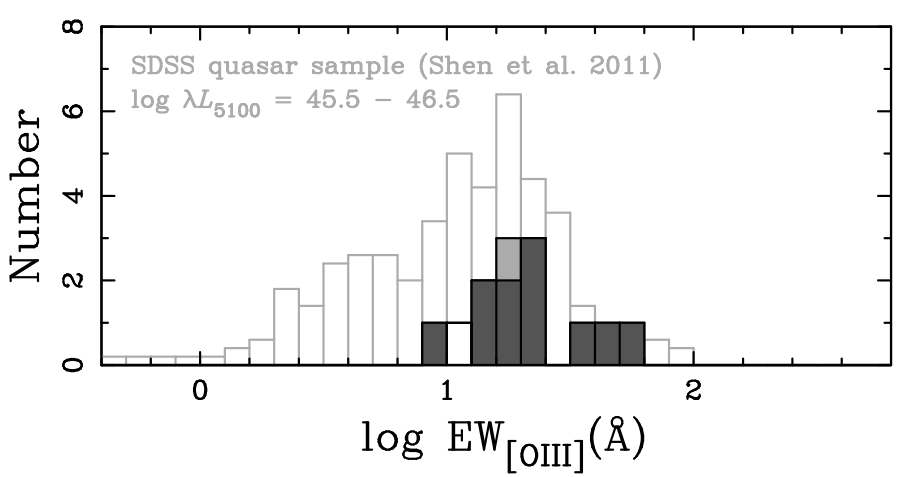

Fig. 3. Distribution of rest-frame equivalent width of [O III] $\lambda 5007$ line. The black line histograms denote our sample of $13 \mathrm{~N}$-loud quasars, the dark gray filled histogram shows our observed $11 \mathrm{~N}$-loud quasars, and the open histogram shows the $3 \sigma$ upper limit. The result of SDSS $\mathrm{J} 1707+6443$ (Araki et al. 2012) is shown as a light gray filled histogram. The gray line open histogram denotes the distribution of 232 SDSS quasars at $0<z<1$ in a luminosity range at $\log \lambda L_{5100}=45.5-$ 46.5 (Shen et al. 2011), which is divided by five to compare with our sample easily.

since some previous studies reported the anti-correlation between the equivalent widths of narrow emission lines and the AGN luminosity (e.g., Steiner 1981; Zhang et al. 2013), for consistency in luminosities between $\mathrm{N}$-loud and general quasars, we selected 232 general quasars at $\log \lambda L_{5100}=45.5-46.5$ from the SDSS sample, which is almost the same range as our N-loud quasar sample. As shown in Fig. 3, we found that the equivalent widths of $\mathrm{N}$-loud quasars are slightly larger than those of general SDSS quasars. The averaged equivalent widths (standard deviations) of N-loud and general quasars are 1.34 (0.22) and 0.99 (0.47), respectively. By using the Kolmogorov-Smirnov (KS) test, we checked that there is no significant difference of their $\mathrm{EW}_{\text {[O III] }}$ distributions $(p=0.004)$.

In addition to measurements of emission lines, we also obtained black hole masses and Eddington ratios of our sample shown in Table 2, estimated based on the FWHM of the $\mathrm{H} \beta$ line and monochromatic luminosity at $5100 \AA$, which is well calibrated in the local Universe (e.g., Vestergaard \& Peterson 2006; Ho \& Kim 2015). Usually, the single-epoch estimation can be 
Table 4. Measurements of rest-frame UV spectra obtained by SDSS observations.

\begin{tabular}{ccccr}
\hline \hline Object & $\begin{array}{c}\log F W H M_{\mathrm{CIV}} \\
{\left[\mathrm{km} \mathrm{s}^{-1}\right]}\end{array}$ & $\begin{array}{c}\log \left(\lambda L_{1350}\right) \\
{\left[\mathrm{erg} \mathrm{s}^{-1}\right]}\end{array}$ & $\begin{array}{c}\log M_{\mathrm{BH}, \mathrm{C} \text { IV }} \\
{\left[M_{\odot}\right]}\end{array}$ & \multicolumn{1}{l}{$\log \left(L / L_{\text {Edd }}\right)_{\mathrm{C} \text { IV }}$} \\
\hline SDSS J0357-0528 & $3.666 \pm 0.045$ & $46.136 \pm 0.011$ & $9.124 \pm 0.040$ & $-0.507 \pm 0.040$ \\
SDSS J0931+3439 & $3.494 \pm 0.143$ & $45.889 \pm 0.019$ & $8.649 \pm 0.125$ & $-0.280 \pm 0.125$ \\
SDSS J1211+0449 & $3.641 \pm 0.286$ & $46.150 \pm 0.013$ & $9.082 \pm 0.249$ & $-0.451 \pm 0.249$ \\
SDSS J0756+2054 & $3.438 \pm 0.163$ & $46.109 \pm 0.017$ & $8.654 \pm 0.142$ & $-0.064 \pm 0.142$ \\
SDSS J0803+1742 & $3.454 \pm 0.039$ & $46.385 \pm 0.008$ & $8.832 \pm 0.034$ & $0.034 \pm 0.034$ \\
SDSS J0857+2636 & $3.447 \pm 0.120$ & $46.422 \pm 0.007$ & $8.838 \pm 0.104$ & $0.065 \pm 0.104$ \\
SDSS J0938+0900 & $3.599 \pm 0.044$ & $46.330 \pm 0.010$ & $9.093 \pm 0.038$ & $-0.283 \pm 0.021$ \\
SDSS J1036+1247 & $3.659 \pm 0.056$ & $46.246 \pm 0.009$ & $9.169 \pm 0.049$ & $-0.442 \pm 0.049$ \\
SDSS J1110+0456 & $3.652 \pm 0.065$ & $46.223 \pm 0.015$ & $9.143 \pm 0.057$ & $-0.439 \pm 0.057$ \\
SDSS J1130+1512 & $3.490 \pm 0.044$ & $46.348 \pm 0.007$ & $8.885 \pm 0.038$ & $-0.057 \pm 0.038$ \\
SDSS J1134+1500 & $3.615 \pm 0.043$ & $46.235 \pm 0.014$ & $9.075 \pm 0.038$ & $-0.359 \pm 0.038$ \\
SDSS J1308+1136 & $3.550 \pm 0.071$ & $46.422 \pm 0.007$ & $9.044 \pm 0.062$ & $-0.141 \pm 0.045$ \\
\hline
\end{tabular}

expressed as follows:

$\log \left(\frac{M_{\mathrm{BH}}}{M_{\odot}}\right)=a+b \log \left(\frac{\lambda L_{\lambda}}{10^{44} \mathrm{erg} \mathrm{s}^{-1}}\right)+c \log \left(\frac{F W H M}{\mathrm{~km} \mathrm{~s}^{-1}}\right)$.

In this study, we adopted a recipe of $\mathrm{H} \beta$-based black hole masses in Vestergaard \& Peterson (2006), i.e., $a=0.91, b=0.50$, and $c=2.00$. Eddington ratios are provided through estimates of bolometric luminosities, using a bolometric correction of $\mathrm{BC}=9.26$ (Richards et al. 2006; Shen et al. 2011). Note that we gave only statistical errors of black hole masses and Eddington ratios in Table 2, excluding systematic ones originating from calibrations and virial parameters. In addition to the near-infrared spectra, our objects have observed optical spectra obtained with SDSS spectrographs (Shen et al. 2008, 2011). Thus, we collected the rest-frame UV measurements, i.e., $\mathrm{FWHM}_{\mathrm{C} \text { IV }}, \log \left(\lambda L_{1350}\right), \log \left(M_{\mathrm{BH}, \mathrm{C} \text { IV }}\right)$, and $\log \left(L / L_{\mathrm{Edd}}\right)_{\mathrm{C} \text { IV }}$, in Table 4. Here, CIV $\lambda 1549$-based black hole masses are estimated with a recipe of $a=0.66, b=0.53$, and $c=2.00$ (Vestergaard \& Peterson 2006). In the estimation of bolometric luminosities from the monochromatic luminosity at $1350 \AA$, we adopted $\mathrm{BC}=3.81$ (Shen et al. 2011). For the $12 \mathrm{~N}$-loud quasars in our sample, measurements of FWHMs of the C IV $\lambda 1549$ line and monochromatic luminosities at $1350 \AA$ are available from Shen et al. (2011).

It is important to note that we ignore the effect of quasar variability on the results for black hole masses and Eddington ratios since a typical luminosity variability amplitude of quasars is only $\sim 0.2$ mag on timescales up to several years (e.g., Sesar et al. 2007; MacLeod et al. 2010, 2012) and the uncertainty of the single-epoch masses would be dominated by measurement errors (see Shen 2013, for more detail).

\section{Discussion}

In this section, we discuss chemical and physical parameters of N-loud quasars by using the measurements as described in Sects. 3 and 4. First, we investigate their NLR metallicities with a diagnostic method consisting of the equivalent width of the [O III] 25007 line (Araki et al. 2012). Second, we discuss the virial black hole mass of $\mathrm{N}$-loud quasars estimated based on the $\mathrm{H} \beta$ line (see Sect. 4). We compare these black hole masses with those based on rest-frame ultraviolet spectra, e.g., the broad C IV $\lambda 1549$ line. We also show their Eddington ratios, and discuss a possible connection between AGN and star formation.

\subsection{NLR metallicities}

The emission line of [O III] $\lambda 5007$ is a collisionally excited line and the emissivity depends strongly on the gas temperature. The equilibrium temperature of ionized gas clouds is significantly affected by gas metallicity because metal emission lines are main coolants of these clouds. Therefore, collisionally excited lines are good indicators of NLR metallicities; if the NLR metallicity is high, these emission lines should be faint.

To see the metallicity dependence of the [O III $] \lambda 5007$ equivalent width for NLR clouds, we used the photoionization code Cloudy (version 13.05; Ferland et al. 1998, 2013). In this calculation, we adopted the same parameters as those in Araki et al. (2012), i.e., the hydrogen densities of $n_{\mathrm{H}}=10^{1.0}$ and $10^{4.0} \mathrm{~cm}^{-3}$ and the ionization parameters of $U=10^{-3.5}$ and $10^{-1.5}$, whose parameter ranges are typical of low- $z$ AGNs: Vaona et al. (2012) determined densities and ionization parameters of local Seyfert galaxies based on optical emission-line diagnostic diagrams (see also Bennert et al. 2006a,b; Nagao et al. 2001, 2002). For the spectral energy distribution (SED) of the photionizing continuum radiation (Mathews \& Ferland 1987), we adopted the "table AGN" command, which reproduces the typical SED of AGNs. For the chemical composition of NLR gas, we assumed that dusty gas clouds contain Orion-type graphite and silicate grains, that all metals are changed with a solar abundance ratio, and we also consider the metal depletion onto dust grains (see Table 7.7 in Hazy, a brief introduction to Cloudy C13.05) except for helium and nitrogen. We used analytical expressions for $\mathrm{He}$ and $\mathrm{N}$ relative abundances as functions of metallicities given in Dopita et al. (2000): for helium, we consider both a primary nucleosynthesis component and the primordial value (Russell \& Dopita 1992) and nitrogen is assumed to be a secondary nucleosynthesis element (e.g., van Zee et al. 1998). The metal depletion is also considered in these elements. We stopped the calculations when the hydrogen ionization fraction dropped below $15 \%$.

Figure 4 shows the simulation result of $\mathrm{EW}_{\left[\mathrm{O}_{\text {III }}\right]}$ as a function of the NLR metallicity. This confirms that equivalent width of the $[\mathrm{O}$ III $] \lambda 5007$ line decreases rapidly as the metallicity increases, which is quite small at $Z_{\mathrm{NLR}}>5 Z_{\odot}$. These results are consistent with the predictions obtained by using the Cloudy version 08.00 in Araki et al. (2012). With this method, we can examine whether NLR metallicities of N-loud quasars are extremely high $\left(Z_{\mathrm{NLR}}>5 Z_{\odot}\right)$ relative to general quasars, although we cannot estimate accurate metallicities only by using the $[\mathrm{O}$ III $] \lambda 5007$ equivalent width. As described in Sect. 4, 


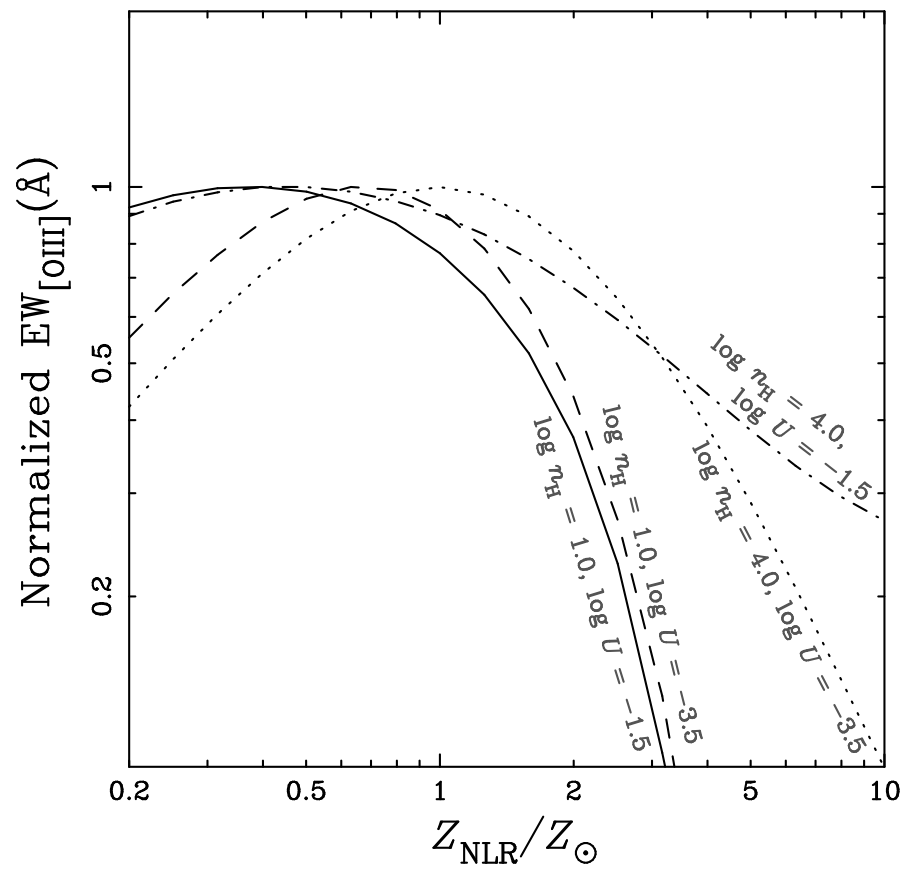

Fig. 4. Normalized equivalent widths of the $[\mathrm{OIII}] \lambda 5007$ line as a function of NLR metallicities, predicted by photoionization models. These are normalized at their respective peaks. Black solid, dashed, dotdashed, and dotted lines are the photoionization model calculations with $\left(\log n_{\mathrm{H}}, \log U\right)=(1.0,-1.5),(1.0,-3.5),(4.0,-1.5)$, and $(4.0,-3.5)$, respectively. Given parameters are also labeled for each line.

we found that there is no significant difference between N-loud quasars and typical SDSS quasars in the same luminosity range. Since NLR metallicities show no significant redshift evolution (Nagao et al. 2006b; Matsuoka et al. 2009) ${ }^{2}$, we are able to compare our sample at $z \sim 2.2$ with the SDSS quasars at $0<z<1$. This result, therefore, indicates that NLR metallicities of N-loud quasars are not extremely high relative to normal ones. This result confirms the result obtained by SDSS J1707+6443 in Araki et al. (2012).

As described in Sect. 4, we also measured $3 \sigma$ flux upper limits of undetected emission lines, i.e., [Ne V] $\lambda 3426$, [O II] $] \lambda 3727$, and $[\mathrm{Ne}$ III] $\lambda 3869$, listed in Table 3 . Thus, to check whether the result obtained by the [O III] $\lambda 5007$ equivalent width is consistent with these upper limits, we compare the observational upper limits with model predictions obtained with the model calculations. Figure 5 shows the emission line flux ratios, i.e., [Ne V] $\lambda 3426 /[\mathrm{O}$ III $] \lambda 5007$, [O II $] \lambda 3727 /[\mathrm{O} \mathrm{III}] \lambda 5007$, and $[\mathrm{Ne}$ III] $\lambda 3869 /[\mathrm{O}$ III] $\lambda 5007$, as a function of NLR metallicities predicted by Cloudy. As shown in Fig. 5a, we find that the predicted emission line flux ratios of $[\mathrm{Ne} \mathrm{V}] \lambda 3426 /[\mathrm{O}$ III $] \lambda 5007$ are below the observational upper limits (gray horizontal lines) for all of the parameter sets examined in our calculations. We note that the predicted $[\mathrm{Ne} \mathrm{V}] \lambda 3426 /[\mathrm{O}$ III $] \lambda 5007$ flux ratio of the models with $\log U=-3.5$ is far below the range shown in Fig. 5a. Figure 5b shows the line flux ratio of

\footnotetext{
2 It has sometimes been reported that the AGN metallicity shows no redshift evolution, which is inferred for BLRs (e.g., Nagao et al. 2006b) and not only for NLRs. Significant redshift evolution of the gas metallicity is reported for star-forming galaxies (e.g., Maiolino et al. 2008), damped Ly $\alpha$ (e.g., Rafelski et al. 2012), and sub-damped Ly $\alpha$ (e.g., Péroux et al. 2003; Som et al. 2015). The origin of the discrepancy in the metallicity evolution between galaxies and AGNs (or the lack of the significant metallicity evolution in AGNs) has been discussed in, e.g., Juarez et al. (2009).
}

[O II $] \lambda 3727 /[\mathrm{O}$ III $] \lambda 5007$ as a function of NLR metallicity; this line flux ratio is sensitive to ionization parameters. This figure implies that it is difficult to explain the observational results with low- $U$ models, i.e., $\log U=-3.5$. On the other hand, models with high ionization parameters satisfy most of observed upper limits at $Z_{\mathrm{NLR}}<4 Z_{\odot}$, whereas at $Z_{\mathrm{NLR}}>5 Z_{\odot}$, the low$n_{\mathrm{H}}$ and high- $U$ model (a solid line) is restricted by all observational upper limits. This is consistent with the result of the [O III] $\lambda 5007$ equivalent width, namely that NLR metallicities of $\mathrm{N}$-loud quasars are not extremely high. Moreover, in Fig. 5c we also confirmed that most objects can be explained with high- $U$ model predictions of [Ne III] $\lambda 3869 /[\mathrm{O}$ III] $\lambda 5007$ ratio instead of low- $U$ models. It seems to be difficult to discuss hydrogen densities with the upper limits. As a result, we confirmed that these observational upper limits are consistent with the scenario that NLR metallicities of N-loud quasars are not extremely high.

In this section, we found that NLR clouds of N-loud quasars are not extremely metal rich generally. To interpret this observational result, we look at two possible scenarios. One is that the metallicity of N-loud quasars is not very high in BLRs and NLRs, assuming the correlation between $Z_{\mathrm{BLR}}$ and $Z_{\mathrm{NLR}}$. In this case, our result indicates that BLR clouds of N-loud quasars are characterized by a very high nitrogen relative abundance without extremely high $Z_{\mathrm{BLR}}$. This means broad emission lines of nitrogen are unreliable indicators of the BLR metallicity in these cases. The second possibility is that $Z_{\mathrm{BLR}}$ is determined by local enrichment at the BLR scale without an effect of global metallicity in their host galaxies traced by NLR metallicities because the total mass of BLR clouds is typically very low $\left(M_{\mathrm{BLR}} \sim 10^{2}-\right.$ $10^{4}$; Baldwin et al. 2003a). This picture can explain the situation that $Z_{\mathrm{NLR}}$ is not very high while $Z_{\mathrm{BLR}}$ is extremely high in $\mathrm{N}$-loud quasars. In this case, since BLR metallicities do not trace chemical properties in Galactic scale, we should use metallicities of NLR gases instead of BLR metallicities if we want to investigate the chemical status in their host galaxies, especially at high redshift. We note, however, that Jiang et al. (2008) shows that emission line flux ratios excluding nitrogen lines of $\mathrm{N}$-loud quasars do not seem to be different from those of normal quasars. This means that BLR metallicities do not have metal-rich gas clouds. Therefore, we conclude that BLR and NLR metallicities of N-loud quasars are not extremely high, and the nitrogen relative abundance is individually as high as in the former scenario. In the following sections we discuss a reason why $\mathrm{N}$-loud quasars show BLR gas clouds containing high $\mathrm{N}$ relative abundance by considering a connection between $\mathrm{BH}$ growth and nitrogen enrichment via star formation.

\subsection{Black hole masses}

As described in Sect. 4, we estimated black hole masses of $\mathrm{N}$-loud quasars based on the rest-frame optical measurements, i.e., FWHM of $\mathrm{H} \beta$ line and continuum luminosities at $5100 \AA$, and the rest-frame UV measurements, i.e., FWHM $\mathrm{C}_{\mathrm{CIV}}$ and $L_{1350}$. In this section, we first examine the relation between $L_{5100}$ and $L_{1350}$, and the $\mathrm{FWHM}_{\mathrm{H} \beta}$ and $\mathrm{FWHM}_{\mathrm{C} \text { IV }}$ relation. Then we compare the black hole masses of $M_{\mathrm{BH}, \mathrm{H} \beta}$ and $M_{\mathrm{BH}, \mathrm{C} \mathrm{IV}}$.

Figure 6 a shows a relation of monochromatic luminosities at 5100 and $1350 \AA$ of N-loud quasars, shown as red circles. For comparison, we also plotted SDSS general quasars at $1.5<$ $z<2.2$ in Shen \& Liu (2012), represented with gray and yellow circles. In the result, we confirmed that $\mathrm{N}$-loud quasars follow the $L_{5100} \AA$ and $L_{1350} \AA$ relation of general quasars. As shown in this figure, our N-loud quasars are distributed below the $1350 \AA$ 
K. Matsuoka et al.: Chemical enrichment and accretion of nitrogen-loud quasars
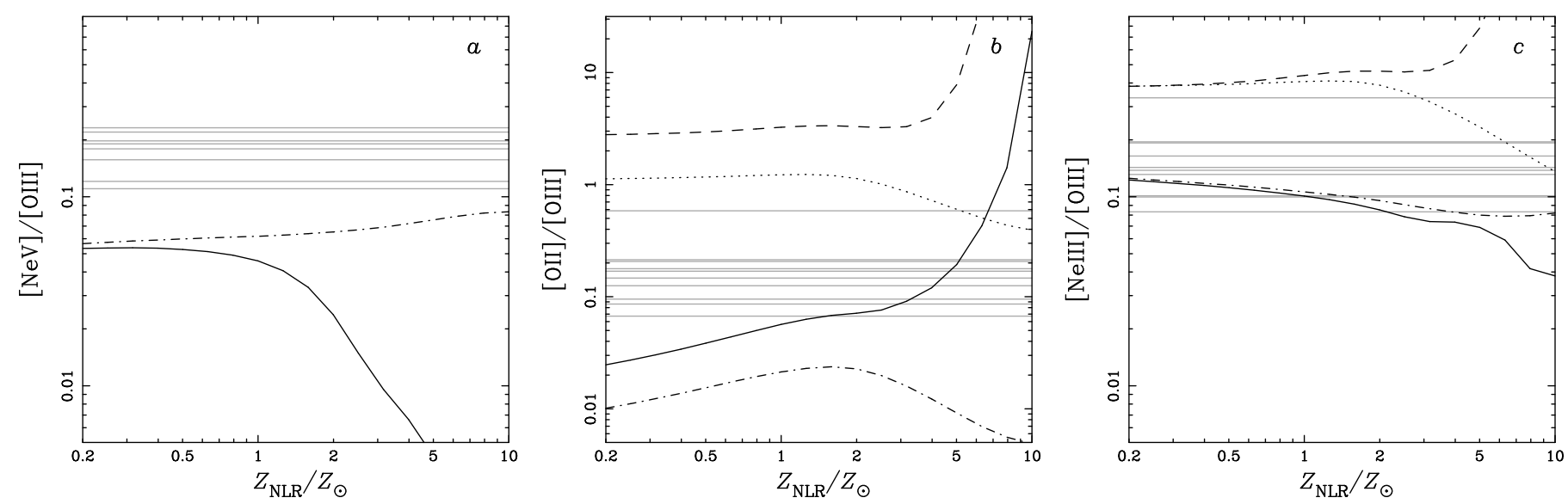

Fig. 5. Model predictions of emission-line flux ratios, i.e., [Ne v] $] 3426 /[\mathrm{O}$ III $] \lambda 5007$ (left, a), [O II $] \lambda 3727 /[\mathrm{O}$ III $] \lambda 5007$ (middle, b), and $[\mathrm{Ne}$ III $] \lambda 3869 /[\mathrm{O} \mathrm{III}] \lambda 5007$ (right, $c$ ), as a function of NLR metallicities. Black solid, dashed, dot-dashed, and dotted lines are the photoionization model calculations with $\left(\log n_{\mathrm{H}}, \log U\right)=(1.0,-1.5),(1.0,-3.5),(4.0,-1.5)$, and $(4.0,-3.5)$, respectively. The left panel shows only two high- $U$ models because [Ne v] 33426 line fluxes in low- $U$ models are fainter than the [O III] $\lambda 5007$ fluxes. Horizontal lines show the $3 \sigma$ upper limits of our sample.
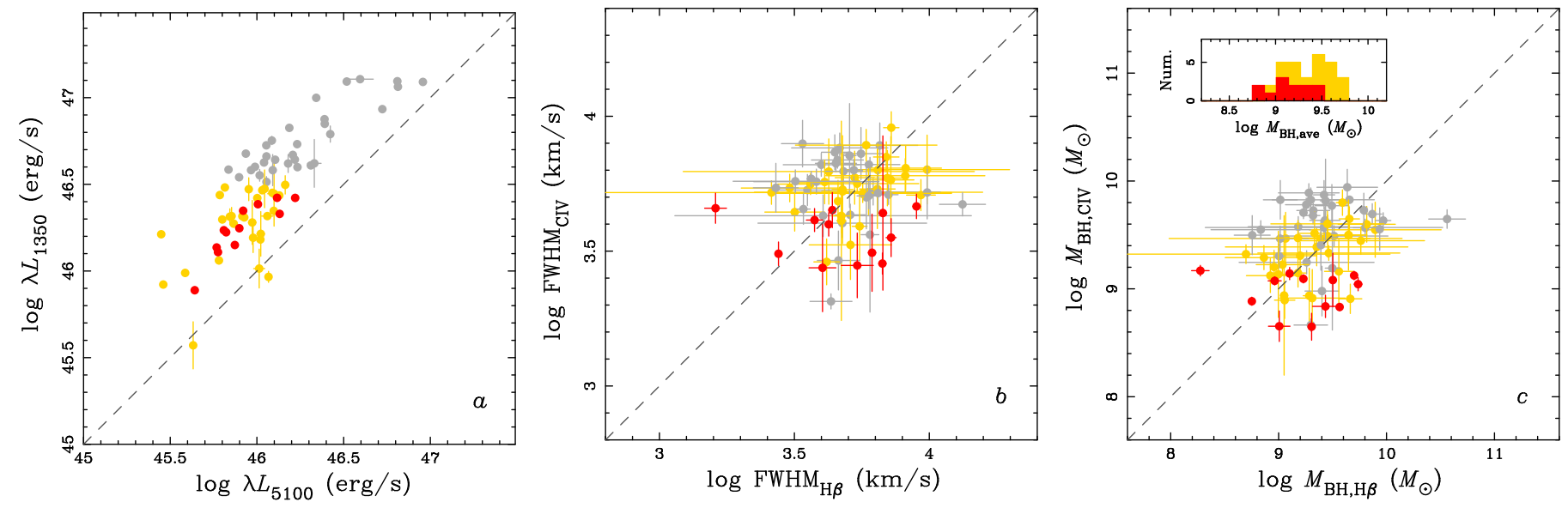

Fig. 6. Comparison of the monochromatic luminosities at $1350 \AA$ and $5100 \AA$ (left, a), the FWHM of the $\mathrm{H} \beta$ line and the C IV $\lambda 1549$ line (middle, $b$ ), and virial black hole masses based on the C IV $\lambda 1549$ line and $\mathrm{H} \beta$ line (right, $c$ ). Our sample of N-loud quasars are shown as red circles. Gray and yellow circles denote SDSS normal quasars at $1.5<z<2.2$ in luminosity ranges of $\log \lambda L_{1350}>46.5$ and $\log \lambda L_{1350}<46.5$ from Shen \& Liu (2012). Errors represent $1 \sigma$ uncertainties of measurements, excluding systematic errors. The one-to-one relations are indicated by dashed lines for each panel.

luminosity of $\log \lambda L_{1350} \sim 46.5$. Thus, to compare them to our $\mathrm{N}$-loud quasars without possible luminosity effects, we colored the quasars at $\log \lambda L_{1350}>46.5$ and $\log \lambda L_{1350}<46.5$ with gray and yellow, respectively. Figure $6 \mathrm{~b}$ shows the comparison of FWHMs of $\mathrm{H} \beta$ and $\mathrm{CIV} \lambda 1549$ lines. It is clear that the FWHM $_{\mathrm{C} \text { IV }}$ of N-loud quasars is typically narrower than those of general quasars, although $\mathrm{FWHM}_{\mathrm{H} \beta}$ seems not to show significant differences between N-loud and normal quasars: the mean values (standard deviations) of $\mathrm{FWMH}_{\mathrm{CIV}}$ and $\mathrm{FWHM}_{\mathrm{H} \beta}$ are $3692 \mathrm{~km} \mathrm{~s}^{-1}\left(715 \mathrm{~km} \mathrm{~s}^{-1}\right)$ and $5159 \mathrm{~km} \mathrm{~s}^{-1}\left(1992 \mathrm{~km} \mathrm{~s}^{-1}\right)$ for $\mathrm{N}$-loud quasars, whereas they are $5507 \mathrm{~km} \mathrm{~s}^{-1}\left(1229 \mathrm{~km} \mathrm{~s}^{-1}\right)$ and $5652 \mathrm{~km} \mathrm{~s}^{-1}\left(1770 \mathrm{~km} \mathrm{~s}^{-1}\right)$ for general quasars. This is consistent with the claim that the line widths of carbon broad emission lines of N-loud quasars are narrower than those of general quasars (Jiang et al. 2008; Bentz et al. 2004). Many studies claimed that highly ionized BLRs with C IV $\lambda 1549$ line are affected by a nonvirial component such as outflow, and would be a biased mass estimator (e.g., Shen \& Liu 2012; Marziani \& Sulentic 2012); however, the $\mathrm{H} \beta$ line widths obtained in this study also have uncertainties due to low $\mathrm{S} / \mathrm{N}$ of near-infrared spectroscopies, implying that black hole masses estimated both with the $\mathrm{H} \beta$ and C IV $\lambda 1549$ lines should involve respective uncertainties. Hence, hereafter, we regard mean black hole masses between them as their representative black hole masses. As shown in Fig. 6c, we found that black hole masses of N-loud quasars seem to be lower than those of general quasars even by focusing on the same luminosity objects shown as yellow circles: the averaged masses are 9.218 and 9.408 logarithmically for N-loud and general quasars, respectively: the averaged masses estimated by $\mathrm{H} \beta$ line are 9.214 and 9.327, and the averaged masses estimated by $\mathrm{C}$ IV $\lambda 1549$ line are 8.965 and 9.320 , respectively. This implies that $\mathrm{N}$-loud quasars are situated in a BH growth phase, although we should keep their large uncertainties of BH masses in mind, and thus we discuss mass accretions into SMBHs with nitrogen enrichment of N-loud quasars.

\subsection{AGN activities of $N$-loud quasars}

In Sect. 5.1, we found that NLR clouds in N-loud quasars are not extremely metal rich, indicating a picture in which only the 


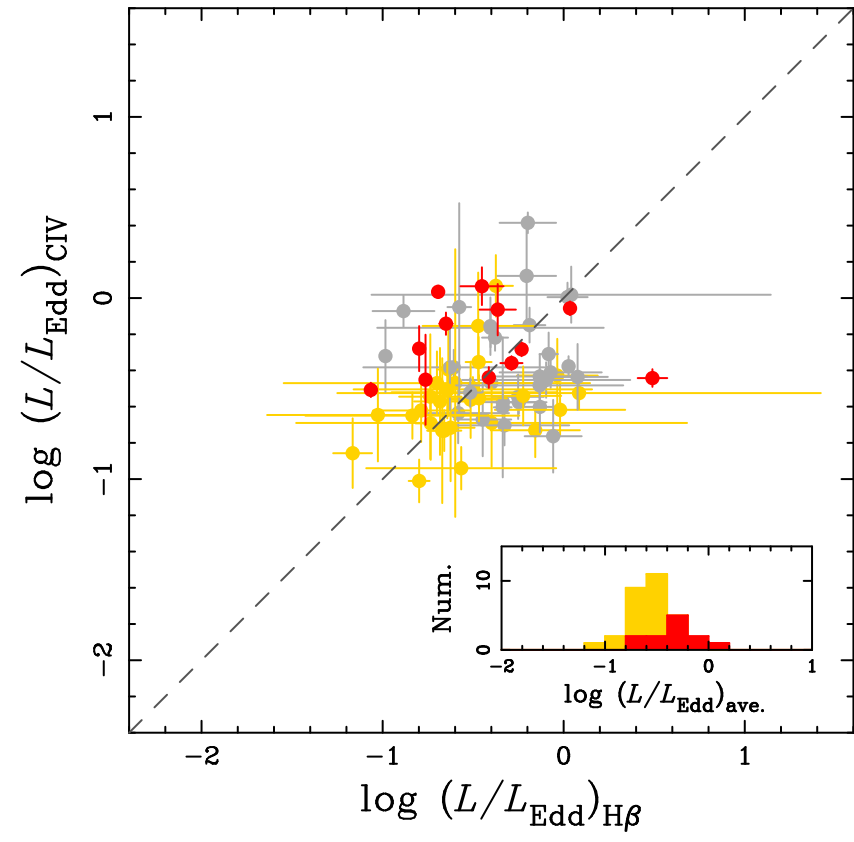

Fig. 7. Comparison of Eddington ratios estimated by the $\mathrm{H} \beta$ line with that by the C IV $\lambda 1549$ line. Red, gray, and yellow circles are the same as those in Fig. 6. The one-to-one relation is indicated by the dashed line. Eddington ratios averaged from $\mathrm{H} \beta$ and $\mathrm{C}$ IV $\lambda 1549$ lines are given as histograms in the small bottom panel. The red- and yellow-filled histograms show averaged Eddington ratios of our N-loud quasars and SDSS normal ones at $\log \lambda L_{1350}<46.5$ from Shen \& Liu (2012), respectively.

nitrogen relative abundance of the BLR gas clouds is relatively high in the N-loud quasars (e.g., Jiang et al. 2008; Araki et al. 2012). In this section, we discuss an interpretation of this nitrogen excess by considering the connection between AGN activity and the nitrogen enrichment due to the nuclear star formation.

Previously, by using optical spectra of 2383 SDSS quasars at $2.3<z<3.0$, we investigated the dependence of the BLR metallicity on the Eddington ratio (Matsuoka et al. 2011) where we utilized emission-line flux ratios of $\mathrm{N} v \lambda 1240 / \mathrm{C}$ IV $\lambda 1549$, N v $\lambda 1240 / H e$ II $\lambda 1640, \quad$ (Si IV $\lambda 1398+O$ IV] $\lambda 1402) / C$ IV $\lambda 1549$, and $\mathrm{AlIII} \lambda 1857 / \mathrm{C}$ IV $\lambda 1549$, which are sensitive to the BLR metallicity. In the result, we found that the Eddington ratio depends on the emission-line flux ratios involving the $\mathrm{N} v \lambda 1240$ line, while it does not correlate with the other emission-line flux ratios, i.e., (Si IV $\lambda 1398+O$ IV] $\lambda 1402) / C$ IV $\lambda 1549$ and Al III $\lambda 1857 / C$ IV $\lambda 1549$ (see Fig. 6 in Matsuoka et al. 2011, for more detail). We suggested that the correlation between the Eddington ratio and the line ratios including nitrogen is tracing a delay of the mass accretion into SMBHs relative to the onset of nuclear star formation of about $10^{8} \mathrm{yr}$ reported by Davies et al. (2007), which is the timescale required for the nitrogen enrichment from AGB stars. In other words, this result indicates that the mass loss from AGB stars enriches BLR gas clouds with nitrogen and enhance the accretion into SMBHs.

By considering the relation between the Eddington ratio and nitrogen abundance described above, we suggest one possible scenario where N-loud quasars are situated in the AGN active phase, i.e., high accretion phase. If this scenario is correct, $\mathrm{N}$-loud quasars should show relatively high Eddington ratios. Thus, we calculated Eddington ratios of $\mathrm{N}$-loud quasars and plotted them in Fig. 7. For comparison, we also plotted the general quasar sample of Shen \& Liu (2012), as in Fig. 6. To examine the distribution of Eddington ratios of N-loud and general quasars, we calculated the averaged Eddington ratios from $\mathrm{H} \beta$-based and C IV 11549 -based black hole masses. The distributions of averaged Eddington ratios of our N-loud sample are shown in Fig. 7 as red-filled histograms. Regarding the general quasars at $\log \lambda L_{1350}<46.5$, we derived averaged Eddington ratios in the same way and shown as the yellow histogram. The mean values (standard deviations) of their Eddington ratios are -0.28 (0.24) and $-0.54(0.22)$ for our N-loud quasars and general ones, respectively. It indicates that Eddington ratios of $\mathrm{N}$-loud quasars are slightly higher than those of SDSS quasars: the KS test confirms the significant difference between their distributions with $p=0.006$. This result means $\mathrm{N}$-loud quasars are significantly situated in a rapid accretion phase, consistent with the scenario that AGB stars enrich BLRs with nitrogen and enhance the accretion into SMBHs.

\section{Conclusions}

We have performed near-infrared spectroscopic observations of $12 \mathrm{~N}$-loud quasars at $2.09<z<2.57$ to investigate their restframe optical properties. We find the following results:

1. The rest-frame optical spectra show that [O III] $\lambda 5007$ equivalent widths of N-loud quasars are slightly larger than those of general quasars, suggesting that NLR metallicities of $\mathrm{N}$-loud quasars are not extremely high, as was expected with strong broad nitrogen lines. If BLR metallicities are related to chemical properties in NLRs, this result indicates a scenario that nitrogen broad lines depend not only on the BLR metallicity, but also on other parameters in some cases.

2. We found N-loud quasars seem to show higher Eddington ratios than those of general quasars, indicating that the $\mathrm{N}$-loud quasars are in a high accretion phase. This result can be predicted from an observational positive correlation between Eddington ratios and nitrogen abundance found in Matsuoka et al. (2011), suggesting a possible connection between AGN activities and star formation where the mass accretion onto SMBH is associated with a post-starburst phase, when AGB stars enrich the interstellar medium with nitrogen.

To understand the chemical properties of high- $z$ galaxies, and also the connection between SMBH growths and galaxy formations, investigating the chemical compositions of BLR gas is crucial. It is especially important to examine whether nitrogen broad lines actually are good indicators of BLR metallicities because they are often used as BLR metallicity indicators. In this sense, understanding the backgrounds of $\mathrm{N}$-loud quasars would be helpful as a first step since N-loud quasars would show us an extreme case regarding chemical evolution and black hole growth.

Acknowledgements. We would like to thank the anonymous referee for the useful comments and suggestions. We are also grateful to Nobuo Araki and Yosuke Minowa for observational support, and we thank the NTT and Subaru telescope staff for their invaluable help during the observations. We would like to thank Gary J. Ferland for providing the great photoionization code Cloudy. K.M. acknowledges financial support from the Japan Society for the Promotion of Science (JSPS) KAKENHI Grant No. 14J01811. T.N. is financially supported by the JGC-S Scholarship Foundation and JSPS KAKENHI Grant Nos. 25707010, 16H01101, and 16H03958. R.M. acknowledges ERC Advanced Grant 695671 "QUENCH" and support by the Science and Technology Facilities Council (STFC). D.P. acknowledges support through the EACOA Fellowship from The East Asian Core Observatories Association, which consists of the National Astronomical Observatories, Chinese Academy of Science (NAOC), the National 
Astronomical Observatory of Japan (NAOJ), Korean Astronomy and Space Science Institute (KASI), and Academia Sinica Institute of Astronomy and Astrophysics (ASIAA). Data analysis were in part carried out on a common-use data analysis computer system at the Astronomy Data Center, ADC, of the National Astronomical Observatory of Japan (NAOJ)

\section{References}

Araki, N., Nagao, T., Matsuoka, K., et al. 2012, A\&A, 543, A143

Bae, H.-J., \& Woo, J.-H. 2014, ApJ, 795, 30

Bae, H.-J., Woo, J.-H., Karouzos, M., et al. 2017, ApJ, 837, 91

Baldwin, J. A., Ferland, G. J., Korista, K. T., Hamann, F., \& Dietrich, M. 2003a ApJ, 582, 590

Baldwin, J. A., Hamann, F., Korista, K. T., et al. 2003b, ApJ, 583, 649

Batra, N. D., \& Baldwin, J. A. 2014, MNRAS, 439, 771

Bennert, N., Jungwiert, B., Komossa, S., Haas, M., \& Chini, R. 2006a, A\&A 456, 953

Bennert, N., Jungwiert, B., Komossa, S., Haas, M., \& Chini, R. 2006b, A\&A, 459,55

Bentz, M. C., Hall, P. B., \& Osmer, P. S. 2004, AJ, 128, 561

Bentz, M. C., Walsh, J. L., Barth, A. J., et al. 2009, ApJ, 705, 199

Davies, R. I., Müller Sánchez, F., Genzel, R., et al. 2007, ApJ, 671, 1388

Dietrich, M., Hamann, F., Shields, J. C., et al. 2003, ApJ, 589, 722

Dietrich, M., Mathur, S., Grupe, D., \& Komossa, S. 2009, ApJ, 696, 1998

Dopita, M. A., Kewley, L. J., Heisler, C. A., \& Sutherland, R. S. 2000, ApJ, 542, 224

Du, P., Wang, J.-M., Hu, C., et al. 2014, MNRAS, 438, 2828

Ferland, G. J., Korista, K. T., Verner, D. A., et al. 1998, PASP, 110, 761

Ferland, G. J., Porter, R. L., van Hoof, P. A. M., et al. 2013, Rev. Mex. Astron. Astrofis., 49, 137

Fu, H., \& Stockton, A. 2007, ApJ, 664, L75

Hamann, F., \& Ferland, G. 1992, ApJ, 391, L53

Hamann, F., \& Ferland, G. 1993, ApJ, 418, 11

Hamann, F., \& Ferland, G. 1999, ARA\&A, 37, 487

Hamann, F., Korista, K. T., Ferland, G. J., Warner, C., \& Baldwin, J. 2002, ApJ, 564,592

Hayano, Y., Takami, H., Guyon, O., et al. 2008, Proc. SPIE, 7015

Hayano, Y., Takami, H., Oya, S., et al. 2010, Proc. SPIE, 7736

Ho, L. C., \& Kim, M. 2015, ApJ, 809, 123

Jiang, L., Fan, X., Vestergaard, M., et al. 2007, AJ, 134, 1150

Jiang, L., Fan, X., \& Vestergaard, M. 2008, ApJ, 679, 962

Juarez, Y., Maiolino, R., Mujica, R., et al. 2009, A\&A, 494, L25

Kewley, L. J., \& Dopita, M. A. 2002, ApJS, 142, 35

Kewley, L. J., \& Ellison, S. L. 2008, ApJ, 681, 1183

Kobayashi, N., Tokunaga, A. T., Terada, H., et al. 2000, Proc. SPIE, 4008, 1056

Kurk, J. D., Walter, F., Fan, X., et al. 2007, ApJ, 669, 32
Lawrence, A., Warren, S. J., Almaini, O., et al. 2007, MNRAS, 379, 1599 Ly, C., Malkan, M. A., Rigby, J. R., \& Nagao, T. 2016, ApJ, 828, 67 MacLeod, C. L., Ivezić, Ž., Kochanek, C. S., et al. 2010, ApJ, 721, 1014 MacLeod, C. L., Ivezić, Ž., Sesar, B., et al. 2012, ApJ, 753, 106

Maiolino, R., Nagao, T., Grazian, A., et al. 2008, A\&A, 488, 463 Marziani, P., \& Sulentic, J. W. 2012, New Astron. Rev., 56, 49 Mathews, W. G., \& Ferland, G. J. 1987, ApJ, 323, 456

Matsuoka, K., Nagao, T., Maiolino, R., et al. 2009, A\&A, 503, 721

Matsuoka, K., Nagao, T., Marconi, A., et al. 2011, A\&A, 527, A100

Moorwood, A., Cuby, J.-G., \& Lidman, C. 1998, The Messenger, 91, 9

Mortlock, D. J., Warren, S. J., Venemans, B. P., et al. 2011, Nature, 474, 616

Nagao, T., Murayama, T., \& Taniguchi, Y. 2001, ApJ, 546, 744

Nagao, T., Murayama, T., Shioya, Y., \& Taniguchi, Y. 2002, ApJ, 575, 721

Nagao, T., Maiolino, R., \& Marconi, A. 2006a, A\&A, 459, 85

Nagao, T., Marconi, A., \& Maiolino, R. 2006b, A\&A, 447, 157

Osmer, P. S. 1980, ApJ, 237, 666

Osmer, P. S., \& Smith, M. G. 1980, ApJS, 42, 333

Pagel, B. E. J., Edmunds, M. G., Blackwell, D. E., Chun, M. S., \& Smith, G. 1979, MNRAS, 189, 95

Park, D., Woo, J.-H., Treu, T., et al. 2012, ApJ, 747, 30

Péroux, C., Dessauges-Zavadsky, M., D’Odorico, S., Kim, T.-S., \& McMahon, R. G. 2003, MNRAS, 345, 480

Rafelski, M., Wolfe, A. M., Prochaska, J. X., Neeleman, M., \& Mendez, A. J. 2012, ApJ, 755, 89

Richards, G. T., Lacy, M., Storrie-Lombardi, L. J., et al. 2006, ApJS, 166, 470

Rupke, D. S., Veilleux, S., \& Sanders, D. B. 2005, ApJ, 632, 751

Russell, S. C., \& Dopita, M. A. 1992, ApJ, 384, 508

Schneider, D. P., Hall, P. B., Richards, G. T., et al. 2007, AJ, 134, 102

Sesar, B., Ivezić, Ž., Lupton, R. H., et al. 2007, AJ, 134, 2236

Shen, Y. 2013, Bull. Astron. Soc. India, 41, 61

Shen, Y., \& Liu, X. 2012, ApJ, 753, 125

Shen, Y., Greene, J. E., Strauss, M. A., et al. 2008, ApJ, 680, 169

Shen, Y., Richards, G. T., Strauss, M. A., et al. 2011, ApJS, 194, 45

Shin, J., Woo, J.-H., Nagao, T., \& Kim, S. C. 2013, ApJ, 763, 58

Som, D., Kulkarni, V. P., Meiring, J., et al. 2015, ApJ, 806, 25

Steiner, J. E. 1981, ApJ, 250, 469

Vanden Berk, D. E., Richards, G. T., Bauer, A., et al. 2001, AJ, 122, 549

van Dokkum, P. G. 2001, PASP, 113, 1420

van Zee, L., Salzer, J. J., \& Haynes, M. P. 1998, ApJ, 497, L1

Vaona, L., Ciroi, S., Di Mille, F., et al. 2012, MNRAS, 427, 1266

Vestergaard, M., \& Peterson, B. M. 2006, ApJ, 641, 689

Warner, C., Hamann, F., \& Dietrich, M. 2004, ApJ, 608, 136

Woo, J.-H., Bae, H.-J., Son, D., \& Karouzos, M. 2016, ApJ, 817, 108

Wu, X.-B., \& Jia, Z. 2010, MNRAS, 406, 1583

Wuyts, E., Wisnioski, E., Fossati, M., et al. 2016, ApJ, 827, 74

York, D. G., Adelman, J., Anderson, J. E., Jr., et al. 2000, AJ, 120, 1579

Zhang, K., Wang, T.-G., Gaskell, C. M., \& Dong, X.-B. 2013, ApJ, 762, 51 